\title{
Evidence of Antioxidant Activity of Novel L-Glutaminase Purified from L. Gasseri BRLHM
}

\author{
${ }^{1}$ Butheina A. Hasson*, ${ }^{2}$ Likaa Hamied Mahdi, ${ }^{3}$ Rajwa Hasen Essa \\ ${ }^{1}$ Division of Biotechnology, Department of Applied Sciences, University of Technology - Iraq \\ ${ }^{2,3}$ Department of Biology, College of Science, Mustansiriyah University - Iraq
}

\section{Article information}

Article history:

Received: July, 03, 2021

Accepted: August, 15, 2021

Available online: December, 04, 2021

Keywords:

Lactobacillus gasseri,

L-glutaminases,

Antioxidant activity

*Corresponding Author:

Butheina A. Hasson

100235@uotechnology.edu.iq

\begin{abstract}
Probiotic strains have the potential to be used as bio-preservatives and functional radical scavenging treatments in the future. Antioxidant tests, including DPPH radical scavenging, were used to evaluate the antioxidant effects of extracellular L- Glutaminase isolated from L. gasseri BRLHM. Parameters for the promoted production of the enzyme under minimal production media were optimized. The importance of this study lies in enhancing the production of the L- Glutaminase isolated from L. gasseri BRLHM with a high activity using these $L$. gasseri bacterial as antioxidant activity. In ion-exchange chromatography, the specific activity was $14.7 \mathrm{U} / \mathrm{mg}$ protein, with $58.8 \%$ yield and 4.6 purification folds. In the gel filtration, the specific activity was $23.4 \mathrm{U} / \mathrm{mg}$ protein, with a yield of $54 \%$ and 4.6 purification folds. According to the findings, L-Glutaminase isolated from $L$.gasseri BRLHM exhibited good antioxidant properties. As the concentration rose, there was a remarkable proportionate increase in scavenging activity. The $\mathrm{IC}_{50}$ values for control and L- Glutaminase were 36.091 .12 and $619.8 \mathrm{gm} / \mathrm{ml}$, respectively. The $\mathrm{IC}_{50}$ values were discovered to be 100 and $200 \mu \mathrm{g} / \mathrm{ml}$, respectively. Conclusion: For the first time, the high of L- Glutaminase isolated L. gasseri BRLHM was shown to exhibit antioxidant. This could be a promising discovery for future radical scavenging treatments and antioxidant prophylaxis with natural probiotics.
\end{abstract}

DOI: 10.53293/jasn.2021.3969.1064, Department of Applied Science, University of Technology

This is an open access article under the CC BY 4.0 License

\section{Introduction}

Probiotics are live microbes that have nutritional and health benefits [1]. Elie Metchnikoff was the first to conduct probiotic research 100 years ago [2]. Lactic acid bacteria (LAB) such as Bifidobacterium sp., Lactobacillus sp., Strep. thermophilus, Enterococcus sp., and Pediococcus acidilactici make up many probiotic microorganisms [3]. Probiotics have several functional qualities that are beneficial to one's health. The study of probiotics' antioxidative capabilities than free radicals is an intriguing research topic. Several human diseases (e.g., inflammatory diseases, cancer, atherosclerosis, and cirrhosis) have been linked to oxidative damage [4]. Damage in DNA was induced by 
oxidative stress. Various malignancies have been found to have higher levels of oxidative DNA damage. Due to the increase of antimicrobial resistance, the inclusion of probiotics in food is a fascinating alternative to antibiotics that have piqued public interest [5]. Antioxidant activity of artificial antioxidants such as butylated hydroxytoluene (BHT), butylated hydroxyanisole (BHA), and n-propyl gallate (PG) is powerful against a variety of oxidation processes. However, due to potential health hazards, the use of these antioxidants in food has been restricted in several countries. Because of the documented negative effects of some artificial antioxidants [6], natural antioxidants are likely to be more tolerable than those created chemically. the result, the hunt for natural, stable, safe antioxidant agents as alternatives to manufactured molecules have gained more attention. The aim of the study is to isolate L-glutaminases from Lactobacillus gasseri and their study antioxidant activity.

\section{Experimental Procedure}

Collection of samples78 vaginal swabs were isolated from healthy women. Lactobacillus spp. is diagnosed based on the findings of culture, microscopic, biochemical, and API $50 \mathrm{CHL}$ testing [7]. Collected of Lactobacillus spp. from aged (15-45) years old from the Hospital AL-elweya of Obstetrics in Baghdad, Iraq, from December 2019 to March 2020. Detection, screening, and purification of L- Glutaminase purified from L. gasseri BRLHM Crude $L$. gasseri prepared by [8], and L-glutaminases was prepared according to [9] with a modification, local isolate $L$. gasseri purified by four steps: ammonium -sulfate precipitation at $80 \%$, dialysis, ion-exchange using SephadexG100 and gel filtration chromatography [10], concentrated of protein by the Bradford method [11] at each step was measured. Study the effectiveness of L-Glutaminase purified from L. gasseri BRLHM as antioxidant.

- DPPH preparation: DPPH is 2,2-Diphenyl-2-picryl-hydroxyl, dissolve (3.3)ml ethanol with (2.3)mg of DPPH. The solution was shielded from light by using aluminum foil to cover the test tubes. Control positive use of ascorbic acid $(10 \mathrm{~g} / \mathrm{ml})$ concentration [12].

- Determination of antioxidant activity of L-Glutaminase by DPPH: Using the free radical scavenging action of L-Glutaminase was investigated [13]. The L-Glutaminase solution (1.0 $\mathrm{mg} / \mathrm{ml})$ was diluted to five different concentrations: 12.5 , $25,50,100$, and $200 \mathrm{mg} / \mathrm{ml}$. Each concentration of L-Glutaminase (10) was mixed with ethanol (490) being and added with a (500) DPPH solution [12]. A residual amount of DPPH was calculated based on a reduction in absorbance at $517 \mathrm{~nm}$ after 30 minutes of incubation at room temperature. The \% inhibition of DPPH was estimated using the equation formula[14], which is shown below.

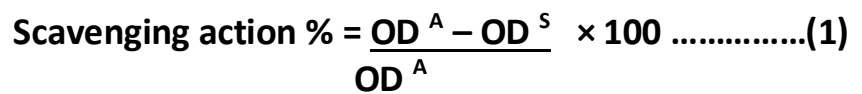

$\mathrm{OD}=$ optical density

$\mathrm{A}=\mathrm{Control}$

$\mathrm{S}=$ Sample 


\section{Results and Discussion}

\subsection{Isolation of Lactobacillus gasseri}

78 isolated go to the Lactobacillus genus were dependent on the microscopic examination, cultural examination, biochemical tests, and API 50 CHL system. L. gasseri and L. crispatus were isolated from vaginal of healthy women's (vaginal swabs') in high percentages $(30.769) \%(\mathrm{n}=24)$ out of 78 Lactobacillus isolates, while $L$. acidophilus constituted (14.102)\% ( $\mathrm{n}=11)$, L. plantarum with $(10.256) \%(\mathrm{n}=8)$, L. casei with $(6.410) \%(\mathrm{n}=5), L$. vaginalis with $(5.128) \%(\mathrm{n}=4)$, and L. jensenii and L. brevis with $(1.282) \%(\mathrm{n}=1)$ out of Lactobacillus isolates.as shown in figure 1.

The vaginal flora was found to be dominated by eight different lactobacilli species, the most common of which were L.crispatus and L. gasseri.

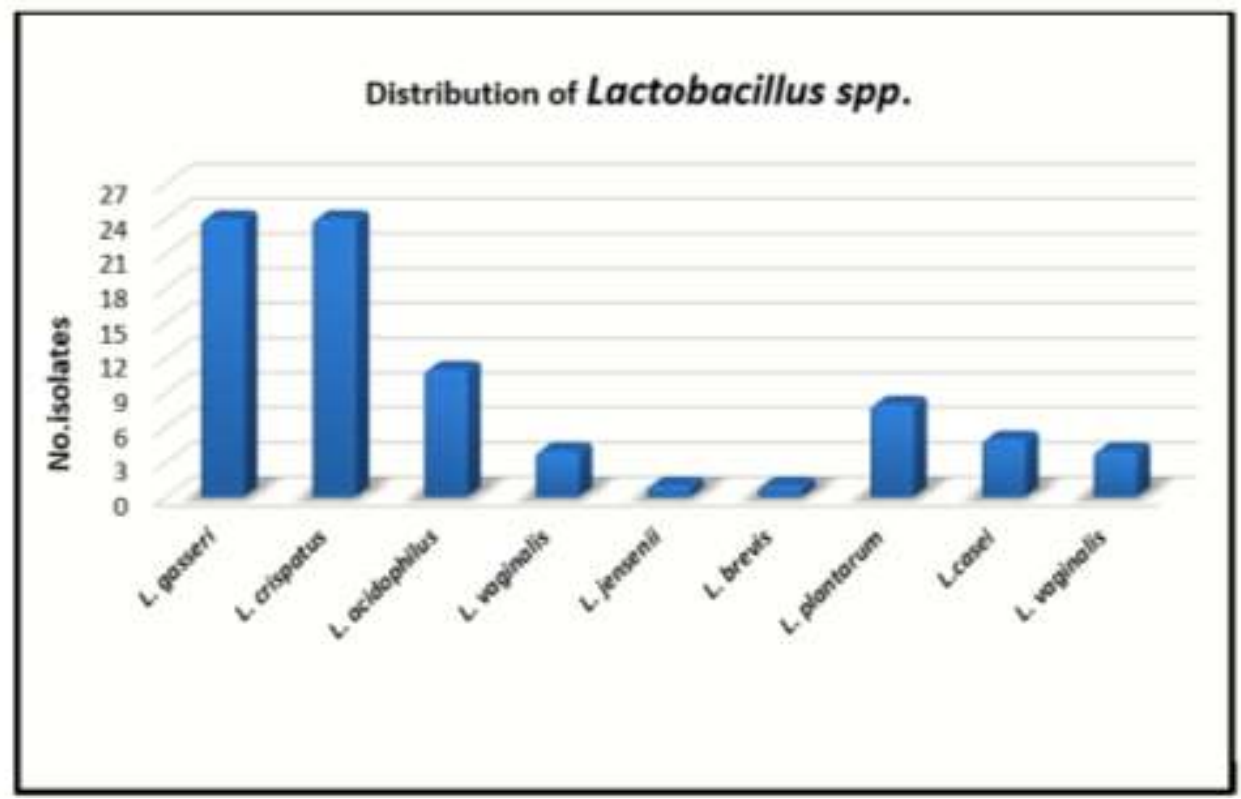

Figure 1: Distribution of Lactobacillus spp. from vaginal of healthy women's

\subsection{Detection of L- Glutaminase purified from L. gasseri BRLHM.}

L. gasseri isolate no.24 was more active in L-Glutaminase production according to the steps of the primary screening for L-Glutaminase production, thus was selected for the following steps of this current study. The selected isolates were characterized by their ability to produce heavy growth L-Glutamine-containing broth medium and had a red or pink color on L-Glutamine broth medium during semi-quantitative screening steps under optimum conditions for L-Glutaminase production, the initial $\mathrm{pH}(7)$, and incubation period (48) $\mathrm{hr}$. at $37^{\circ} \mathrm{C}$. The L-Glutaminase activity indicated that the L. gasseri and specific activity reached 5 Unit/mg proteins.

\subsection{Semi-Quantitative Screening}

The L. gasseri BRLHM isolate no.24 isolate was cultured on an L-Glutamine broth medium containing LGlutamine and monitored during $48 \mathrm{hr}$. of incubation, resulted from the reaction of ethanolic phenol red due to the variation in ammonia production between bacterial isolates since the active L- Glutaminase producing isolates 
increased NH3 and intensity of color production which indicated the activity of this L-Glutaminase screening method Figure 2. The 12 L. gasseri BRLHM isolates that grow on L-Glutamine broth during $24 \mathrm{hr}$. revealed pink color, while the other isolates formed pale yellowish color. The color intensity indicated bacterial activity to hydrolyze L-Glutamine and liberate glutamic acid and ammonia; this can be used as an indicator to produce Lglutamine. L.gasseri BRLHM isolate no.24 isolate was selected for further steps of this current study. This color change is due to changes in the $\mathrm{pH}$ of the medium, as L-Glutaminase causes the breakdown of the amide bond in L-Glutamine and liberates ammonia the L- Glutaminase activity present in Lactobacillus. Lactobacillus screening is based on the qualitative approach that Gulati et al., [20] identified. The change of color of the medium from yellow to pink is an indicator of the extracellular production of L- Glutaminase. This color shift is attributable to a change in the $\mathrm{pH}$ of the medium. Phenol red is yellow at acidic $\mathrm{pH}$ and turns pink at alkaline $\mathrm{pH}$, thereby forming a pink zone around the bacterial colonies. The findings were those in agreement with (Savitha et al., [21].

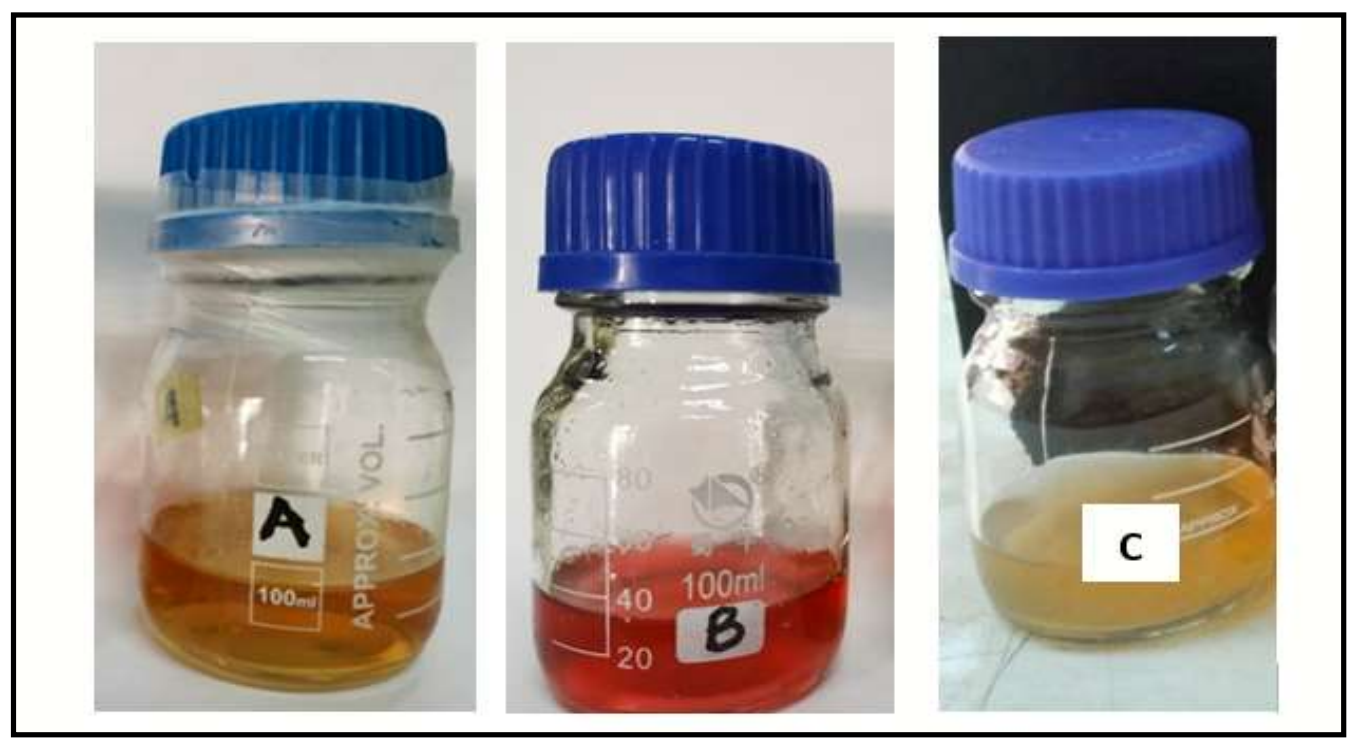

Figure 2: L- Glutaminase production on L-Glutamine broth medium during $48 \mathrm{hr} . \mathrm{A}=$ control, $\mathrm{B}=$ strong pink, $\mathrm{C}=$ pale pink weakly.

\subsection{L-glutaminases Purification}

Dialysis, Ion exchange technique using Sephadex-G100 column, with $80 \%$ ammonium sulfate precipitation saturation have been used to L-glutaminases purification was eluted using a linear gradient of $0.05-0.1 \mathrm{M} \mathrm{NaCl}$. The first peak was found between 0.05 and $0.1 \mathrm{M}$, while the second peak was L-glutaminases, which eluted in $0.4-0.5 \mathrm{M} \mathrm{NaCl}$. L-glutaminases that eluted in 5-10\% of $1 \mathrm{M} \mathrm{NaCl}$, and L-glutaminases that eluted in $40-50 \%$ of $1 \mathrm{M} \mathrm{NaCl}$, are the first and second peaks, respectively. In ion-exchange chromatography, the specific activity of L-glutaminases was $14.7 \mathrm{U} / \mathrm{mg}$ protein, with $58.8 \%$ yield and 4.6 purification folds. figure. 3 . In the gel filtration the specific activity of L-glutaminase was $23.4 \mathrm{U} / \mathrm{mg}$ protein, with a yield of 54 percent and 4.6 purification folds. figure 4. This study introduced a new L-glutaminase which belonged to the 1-glutaminases group and was termed L-glutaminase BRLHM because it differed from other L-glutaminase identified by lactobacillus. Purification of L- Glutaminase was critical for gaining a better knowledge of the enzyme's function [15]. 


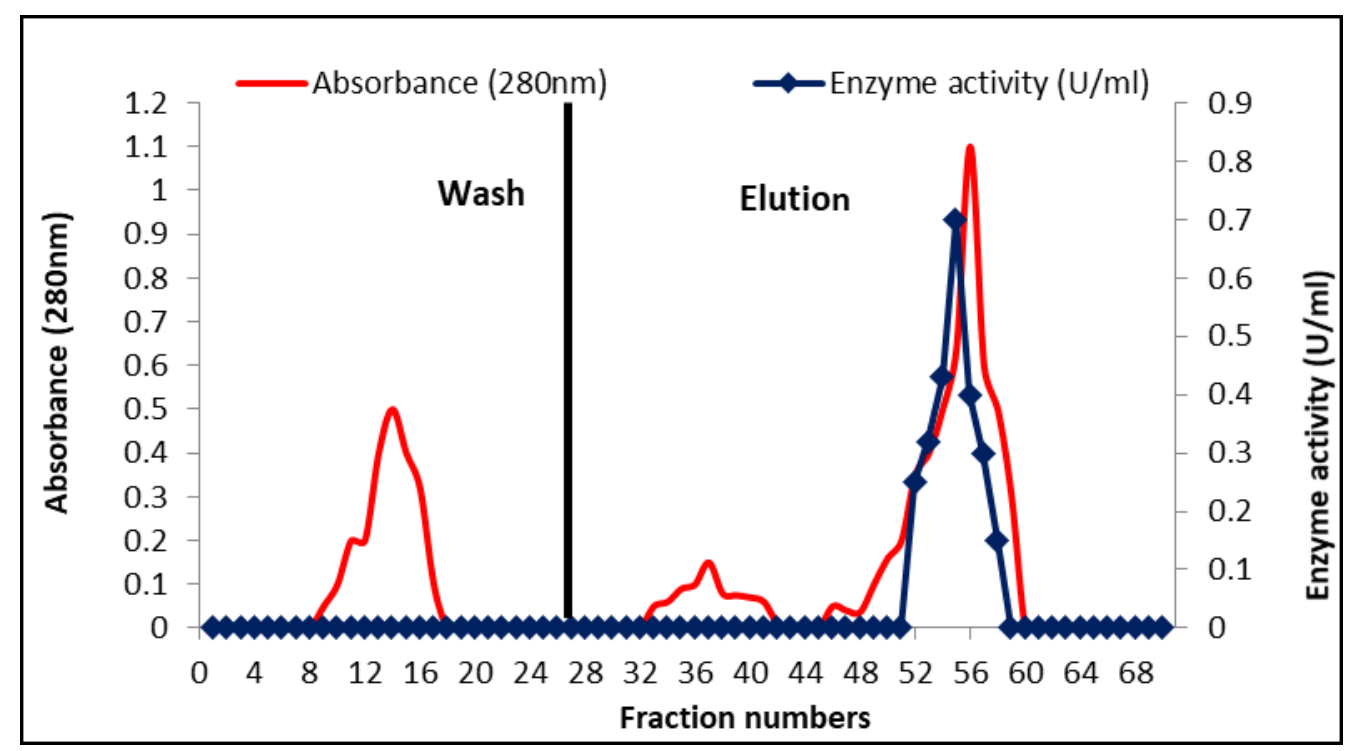

Figure 3: Ion exchange chromatography for L- Glutaminase.

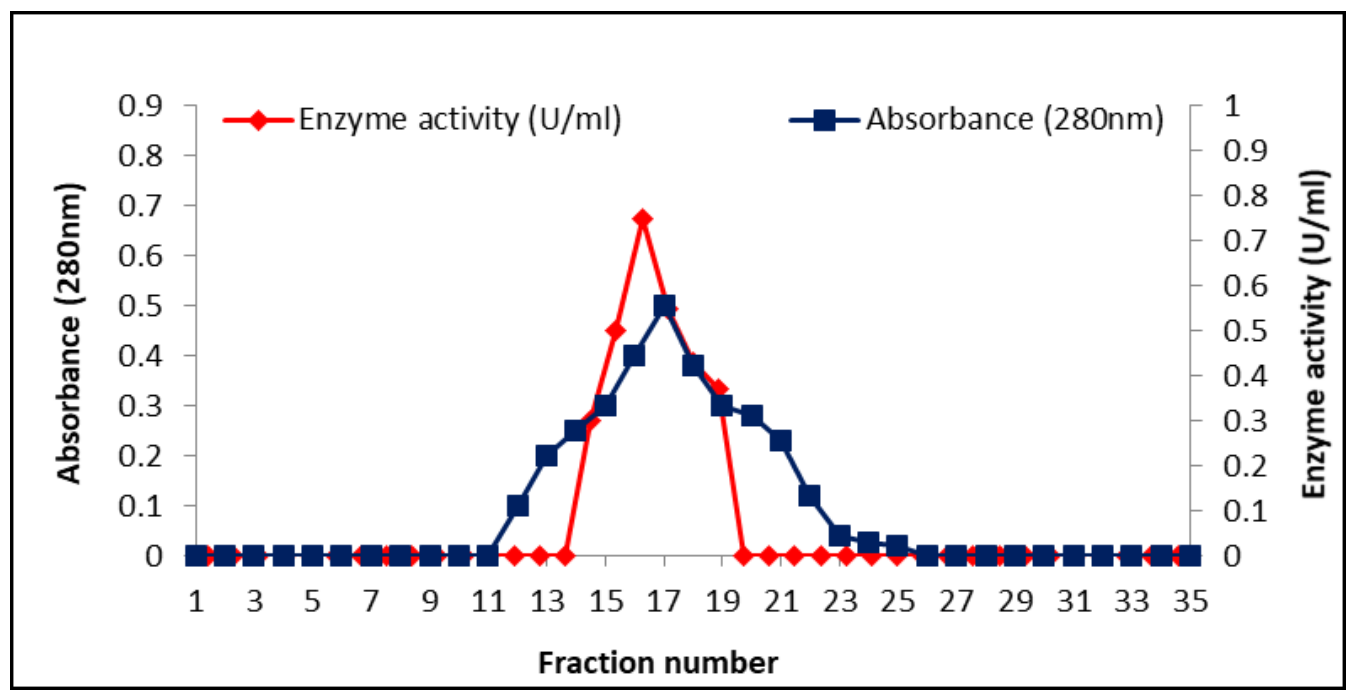

Figure 4: Gel filtration chromatography for L- Glutaminase.

\subsection{Antioxidant activity of L- Glutaminase by using DPPH}

The results of my study of L- Glutaminase purified from L. gasseri BRLHM isolate showed the DPPH root was displaced immediately proportional to the increase in concentration. The scavenged effect is assigned a concentration of $(12.5,25,50,100$, and 200$) \mathrm{g} / \mathrm{ml}$, as shown in figure 5. P-value test ascorbic acid was $<0.0012$ at concentration $200 \mu \mathrm{g} / \mathrm{ml}$. while the P-value test for purified L-Glutaminase was $<0.0001$ at a concentration of 200 $\mu \mathrm{g} / \mathrm{ml}$. The $\mathrm{IC}_{50}$ value of control and L- Glutaminase purified from L. gasseri BRLHM isolate are (36.09 \pm 1.12 and $619.8 \mu \mathrm{g} / \mathrm{ml}$ respectively. For DPPH radicals, the $\mathrm{IC}_{50}$ values were determined to be 100 and $200 \mathrm{~g} / \mathrm{ml}$, respectively. as indicated in table (1). The intact cells of L- Glutaminase purified L. gasseri isolate displayed powerful antioxidant activity in vitro, which was like the antioxidant activity of $L$.plantarum by [16]. These findings backed with confirmed of [17], who found that proteins isolated from Bifidobacterium animalis cells have antioxidant activity in vitro. 


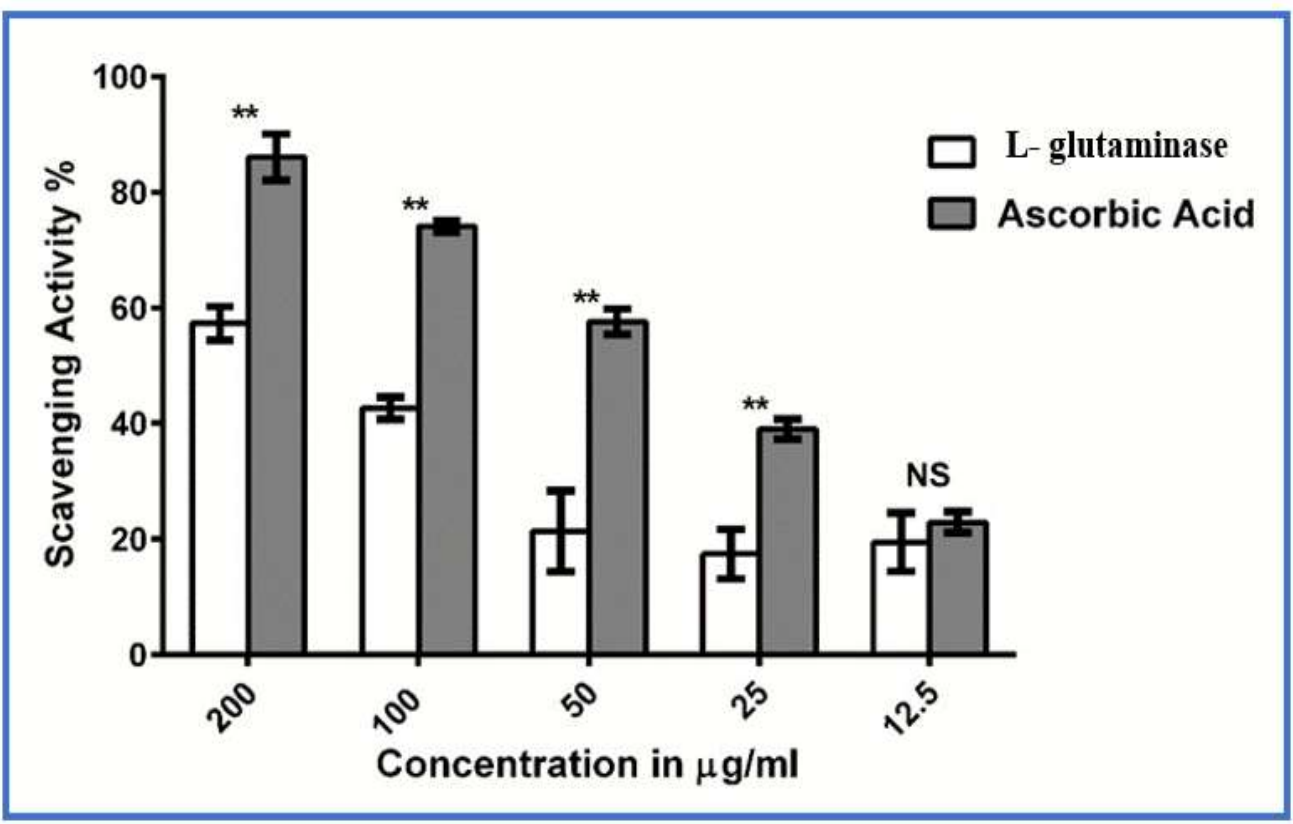

Figure 5: Antioxidant activity of L- Glutaminase purified from L. gasseri BRLHM isolate by DPPH.

One of the most often utilized tests is the scavenged effect test. Pure L-Glutaminases and a standardized antioxidant molecule are used to demonstrate the DPPH radical scavenging capability of purified L-Glutaminases. When antioxidant compounds returned an electron and a proton, the hue shifted to yellow [14].

Table 1. IC 50 value for antioxidant activity of L- Glutaminase

\begin{tabular}{|c|c|c|}
\hline Samples & $\begin{array}{c}\text { IC50 value in } \mu \mathrm{g} / \mathrm{ml} \text { (DPPH free } \\
\text { radical) }\end{array}$ & P value \\
\hline L-Glutaminase & 619.8 & $<0.0001$ \\
\hline Control & $36.09 \pm 1.12$ & $<0.0012$ \\
\hline
\end{tabular}

Glutamine can influence redox homeostasis, bioenergetics, nitrogen balance, and other processes, being a precursor to glutathione, the most important non-enzymatic cellular antioxidant, and it is convert glutamine to glutamate, which is then converted to alpha-ketoglutarate for further metabolism [18]. L-Glutaminase utilized scavenge free radicals generated in vitro by donating their $\mathrm{H}$, which is the most widely used technique for evaluating the antioxidant capacity of pure substances. As a result, discovering antioxidants from natural sources is becoming increasingly popular [19]. 


\section{Conclusion}

My study demonstrated for the first time that the high-level of L- Glutaminase was purified L. gasseri BRLHM to have antioxidant effects. This could be a promising discovery for future radical scavenging therapies and antioxidant prophylaxis with natural probiotics.

\section{Acknowledgments}

The authors thank the department Biotechnology/University of Technology and College of Science/AlMustansiriyah and for funding the activities presented in this study.

Conflicts of interest: There are no conflicts of interest.

\section{References}

[1] W.P. Charteris, P.M. Kelly, L. Morelli, J.K. Collins, et al., "Development and application of an in vitro methodology to determine the transit tolerance of potentially probiotic Lactobacillus and Bifidobacterium species in the upper human gastrointestinal trac," Journal of Applied Microbiol, vol. 84, no. 12, p. 759768,1998 .

[2] I. Muhammad, A.A. Ahmad, and T.Shah, "Health promoting and disease preventing properties of probiotics with special reference to lactobacillus," A review Journal Probiotic Health, vol. 6. no.10,p.2329-8901,2018.

[3] R.Sharma, B.Bhaskar,B. Sanodiya,G.Thakur, P. Jaiswal, et al., "Probiotic Efficacy and Potential of Streptococcus thermophilus modulating human health," A synoptic review. IOSR Journal Pharmaceutical Biology Sciences, vol.9,p. 52-58,2014.

[4] A.G.Abdel-Razek, A.N.Badr, G.S.Mohamed, et al., "Characterization of olive oil By-products: antioxidant activity, its ability to reduce aflatoxigenic fungi hazard and its aflatoxins,"Annul Research Reviewer Biology, vol.14, p. 1-14, 2017.

[5] J.Cleveland, T.Montville, I.Nes, M.Chikindas, et al., "Bacteriocins: Safe, natural antimicrobials for food preservation,” International Journal Food Microbiology, vol.71, p. 1-20, 2001.

[6] B.Sah, P.Vasiljevic, T. McKechnie, and O. Donkor, "Effect of probiotics on antioxidant and antimutagenic activities of crude peptide extract from yogurt," Food Chemechal, vol.156, p. 264-270, 2014.

[7] R. Jabbar and N.N. Hussein, "Evaluation the Antibacterial Activity of Biosynthesis Silver Nanoparticles by Lactobacillus Gasseri Bacteria," Journal of Applied Sciences and Nanotechnology, vol.1, no.3, p.86-95, 2021.

[8] J.C.De Man, D.N. Rogosa, and M.E.Sharpe, "A medium for the cultivation of lactobacilli," Journal applied Bacteriology, vol.23, no.1, p.130-135, 1960.

[9] A.Imada, S.Igarasi,K. Nakahama, M.Isono, "Asparaginase and glutaminase activities of microorganisms," Journal Gene Microbiology, vol. 76,p. 85-99,1973.

[10] K.Pandian, V. Deepak, D. Sivasubramaniam, H.Nellaiah, K.Sundar, et al., "Optimization and purification of anticancer enzyme L-glutaminase from Alcaligenes faecalis KLU102," Biology Journal, vol.69, no.12, p.1644-1651, 2014.

[11] M.M.Bradford. "A rapid and sensitive method for the quantitation of microgram quantities of protein utilizing the principle of protein-dye binding," Annals Biochemical, vol. 72, p. 248-54, 1976.

[12] K.M.Raghad,I.J. Ahmed, H.A.Butheina ,H.N. Nehia et al., "Preparation and characterization of graphene oxide for biological application," Drug Invention Today,vol.14,no.5,p1-7,2020. 
[13] J.H.Kareem,H.S.Tahreer, and H.A. Buthenia, "Preparation of Aluminum Oxide Nanoparticles by Laser Ablation and a Study of Their Applications as Antibacterial and Wounds Healing Agent," Nano Biomedicine Engineering, vol. 11, p.313-319, 2019.

[14] J.H.Kareem, H.A.Buthenia, and H.N.Nehia, "Biological application of titanium dioxide nanoparticles prepared through laser ablation in liquid," Drug Invention Today, vol.12, p. 10-16, 2019.

[15] A.Ayodeji , S.Suren, and P. Santhosh,"Recent advances in microbial glutaminase production and applications-a concise review," Critical Review Biotechnology, vol.3, p. 1549-7801, 2019.

[16] S. Li, Y. Zhao, L.Zhang,X. Zhang,L.Huang, et al ., "Antioxidant activity of Lactobacillus plantarum strains isolated from traditional Chinese fermented foods," Food Chemical, no.135, p. 1914-1919, 2012.

[17] Q.Shen, N.Shang, and P.Li , “ In vitro and in vivo antioxidant activity of Bifidobacterium animalis 01 isolated from centenarians," Current Microbiology, vol.62,p. 1097-1103,2011.

[18] K.Jambulingam , and S.Sudhakar, "Purification and characterization of a novel broad spectrum anti-tumor L-glutaminase enzyme from marine Bacillus subtilis strain JK-79," African Journal of Microbiology Research. Vol.13, no.12, p.232-244, 2019.

[19] Y.Gülçin. "Antioxidant activity of 1-adrenaline: A structure-activity insight," Chemical Biology. Interactional, vol. 179, p. 71-80, 2009.

[20] R.Gulati, J.Saxena, and R.Gupta, "A rapid plate assay for screening L-asparaginase producing microorganisms," Letters in Applied Microbiology, vol.24, p. 23-26, 1997.

[21] S.Savitha ,N. Desai, J.Sonal, J. Chopra, S.Basavaraj,F. Hungund.et al.,"Production, purification and characterization of L-Glutaminase from Streptomyces sp. isolated from soil," Journal of Applied Pharmaceutical Science, vol. 6, no.07, p. 100-105,2016. 